\title{
Neurofeedback to improve neurocognitive functioning of children treated for a brain tumor: design of a randomized controlled double-blind trial
}

Marieke A de Ruiter ${ }^{1 *}$, Antoinette YN Schouten-Van Meeteren², Rosa van Mourik ${ }^{3}$, Tieme WP Janssen ${ }^{3}$, Juliette EM Greidanus ${ }^{1}$, Jaap Oosterlaan ${ }^{3}$ and Martha A Grootenhuis ${ }^{1}$

\begin{abstract}
Background: Neurotoxicity caused by treatment for a brain tumor is a major cause of neurocognitive decline in survivors. Studies have shown that neurofeedback may enhance neurocognitive functioning. This paper describes the protocol of the PRISMA study, a randomized controlled trial to investigate the efficacy of neurofeedback to improve neurocognitive functioning in children treated for a brain tumor.

Methods/Design: Efficacy of neurofeedback will be compared to placebo training in a randomized controlled double-blind trial. A total of 70 brain tumor survivors in the age range of 8 to 18 years will be recruited. Inclusion also requires caregiver-reported neurocognitive problems and being off treatment for more than two years. A group of 35 healthy siblings will be included as the control group. On the basis of a qEEG patients will be assigned to one of three treatment protocols. Thereafter patients will be randomized to receive either neurofeedback training $(n=35)$ or placebo training $(n=35)$. Neurocognitive tests, and questionnaires administered to the patient, caregivers, and teacher, will be used to evaluate pre- and post-intervention functioning, as well as at 6-month follow-up. Siblings will be administered the same tests and questionnaires once.
\end{abstract}

Discussion: If neurofeedback proves to be effective for pediatric brain tumor survivors, this can be a valuable addition to the scarce interventions available to improve neurocognitive and psychosocial functioning.

Trial registration: ClinicalTrials.gov NCT00961922.

Keywords: Brain tumor, Child, Survivors, Attention, Memory, Processing speed, Neurocognitive functioning, Intervention, Neurofeedback, Protocol, RCT, Double-blind

\section{Background}

As a result of improved treatment, the survival rate of children diagnosed with a brain tumor has increased considerably [1]. As a consequence, neurocognitive longterm effects of the tumor and the treatment are reported more often, including deficits in attention, processing speed, and memory [2-4]. Radiotherapy, chemotherapy, tumor location, and longer time since diagnosis are related to worse neurocognitive functioning $[5,6]$. A

\footnotetext{
*Correspondence: m.a.deruiter@amc.nl

'Psychosocial Department, Emma Children's Hospital AMC, room A3-241, Meibergdreef 9, Amsterdam 1105 AZ, The Netherlands

Full list of author information is available at the end of the article
}

major consequence of these impairments is the decline in ability to acquire new skills and information, which leads to an increasing gap in the development between patients and their peers. This, in turn, has its impact on educational results, vocational success and may compromise social competence and quality of life [7].

Butler and Mulhern have emphasized that interventions should be developed to improve neurocognitive functioning and subsequently improve future perspectives of these children [8]. Interventions that are considered relevant for survivors with cancer-related brain injury are cognitive remediation and pharmacotherapy $[9,10]$. A cognitive remediation program, using techniques from

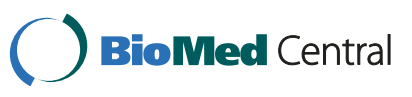


three disciplines: brain injury rehabilitation, special education and clinical psychology, has been developed and tested by Butler and colleagues [9]. Participants in the randomized controlled trial were 161 survivors of a childhood cancer, whose malignancy and/or treatment involved the central nervous system. The results showed improvements in caregiver reported attention and academic achievement, although the effect sizes were modest. Van 't Hooft et al. have investigated the effects of a cognitive training program on neurocognitive function with a randomized controlled trial, enrolling 38 patients with acquired brain injury, including 14 brain tumor survivors [10]. The training program consisted of memory and attention exercises, in combination with cognitive behavioral training. The children in the treatment group showed sustained positive effects on memory and attention functioning until six months after the training, but not on processing speed.

Regarding pharmacotherapy, it has been suggested that survivors of childhood cancer may benefit from stimulant medication as used in the treatment of attention deficit hyperactivity disorder (ADHD). Attention deficits in survivors of brain tumors are likely to improve by methylphenidate. Mulhern and colleagues found improvements of attention in 37 long term survivors of a malignant brain tumor after methylphenidate [11]. In a randomized placebo-controlled double-blinded trial including 32 survivors of a brain tumor $(n=25)$ or acute lymphoblastic leukemia $(n=7)$, Thompson et al. found that methylphenidate led to improved sustained attention [12]. A drawback of pharmacotherapy is the possibility of side effects, e.g. sleep disturbance, weight loss, anxiety, and sadness [13]. Also, this medication does not lead to a sustained effect unless the patient continues the pharmacotherapy.

The limited current available options warrant the search for alternatives. Neurofeedback is a relatively new form of therapy, which has never been investigated in pediatric brain tumor survivors. Neurofeedback is a behavioral intervention that is based on the principles of operant conditioning. During the therapy the patient is presented with real-time feedback on his or her brainwaves, as measured by one or more electrodes on the scalp. The patient is reinforced when the brain produces a certain desired wave. Reinforcement may comprise seeing a movie or hearing music. The desired brain wave is determined by a quantified electro encephalogram (qEEG), which is conducted prior to the training.

The effects of neurofeedback have been discovered serendipitously by Sterman, when cats having received feedback of $12-15 \mathrm{~Hz}$ on the motor cortex showed to be less susceptible to epileptic seizures [14]. There is a large body of scientific research documenting the effectiveness of neurofeedback for the treatment of diverse pathological conditions as summarized in comprehensive reviews, including ADHD, traumatic brain injury and schizophrenia [15-19].

Strehl et al. showed that children with ADHD were able to learn to regulate their brain activity by neurofeedback [20]. After training, significant improvements in behavior, attention, and IQ scores were found. All changes proved to be stable at six months follow-up after the end of training. Hodgson et al. conclude in their meta-analysis on nonpharmacological interventions for ADHD that neurofeedback resulted in significant improvements of DSM-IV symptoms of ADHD, neurocognitive functioning and behavior [18]. In a comparative study researchers found that the positive effects of neurofeedback for children with ADHD were superior to a computerized attention training at six months follow up [21]. However, to date there is a lack of published studies that employ a randomized placebocontrolled double-blind design when investigating neurofeedback [22].

Brain tumor survivors differ from ADHD patients, as they have structural brain damage caused by the tumor, surgery, radiotherapy and/or chemotherapy. An indication that neurofeedback might be effective in pediatric brain tumor survivors may be derived from the results of studies into the effects of neurofeedback in patients with traumatic brain injury. A review of Thornton and colleagues [23] describes a total of 44 studies (12 RCT, 16 comparative, 16 correlation) with traumatic brain injury patients reporting improved attention, cognitive flexibility, cognitive performance, and problem solving after neurofeedback, providing strong initial support for the idea that neurofeedback could be used in patients with structural brain damage. Subsequently, Aukema and colleagues conducted a pilot study into the feasibility of neurofeedback on 9 brain tumor survivors in our hospital [24]. This study demonstrated that it was feasible to use neurofeedback with brain tumor survivors. All participants completed the training and were positive about the training they received, as they would recommend it to others. Patients reported decreased subjective fatigue after the training. Also the test results showed that processing speed improved in 6 out of 9 patients. These findings warranted the set up of a larger study into the effectiveness of neurofeedback for pediatric brain tumor survivors.

The current paper describes the protocol of the PRISMA study (pediatric research on improving speed, memory, and attention); a randomized controlled double-blind trial, approved by the medical ethical committee of the Academic Medical Centre in Amsterdam. The primary aim of the PRISMA study is to investigate the efficacy of neurofeedback for improving neurocognitive functioning after treatment for a pediatric brain tumor. Secondary, we hypothesize that subsequent to the expected 
neurocognitive changes achieved with neurofeedback, children will experience improved psychosocial functioning [25]. Neurocognitive functioning will be investigated by tests administered to the patient. Psychosocial functioning will be measured using patient-reported as well as caregiver and teacher reported questionnaires. Assessments will take place pre and post training, as well as six months post training, in order to examine the longterm effects of the training. Comparing the effects of neurofeedback to placebo feedback will assess efficacy of neurofeedback. Pre training results obtained with the brain tumor survivors will be compared to a control group of healthy siblings, to assess the level of dysfunction on the measures used in this study.

\section{Methods}

\section{Study design}

This study is a randomized placebo-controlled doubleblind trial, to investigate whether neurofeedback improves neurocognitive functioning in children who have received treatment for a brain tumor (trial number clinicaltrials.gov NCT00961922). After enrollment, patients will be randomized into two groups: (1a) the experimental group, receiving neurofeedback, and (1b) the placebo group, receiving placebo training. In addition, (2) a control group of healthy siblings is included; this group will not receive any training. If effectiveness of neurofeedback is demonstrated after completion of the study, patients in the placebo group will be given the opportunity to receive neurofeedback.

\section{Participants}

Eligible for inclusion are patients in the Netherlands, aged 8 to 18 years, who finished treatment for a brain tumor at least two years prior to enrolment and who suffer from problems in neurocognitive functioning. Problems in neurocognitive functioning include attention problems, problems with information processing speed and/or memory problems as assessed by caregiver report. Exclusion criteria are premorbid diagnosis of ADHD or ADD, a mental or physical condition that prohibits neurocognitive assessment and insufficient mastery of the Dutch language. Siblings, aged between 8 and 18 years, form the control group.

\section{Intervention}

The neurofeedback training is performed at home or school using a Dell notebook (Inspiron N5030, 15.6 inch screen), with BioExplorer software, version 1.5 installed, and a portable Brainquiry PET neurofeedback device $[26,27]$. Reinforcement is provided by a self-selected movie that will be presented on the screen if the brain produces the desired activity, as detected by an electrode placed at $\mathrm{Cz}$ (see Figure 1). Each patient receives two

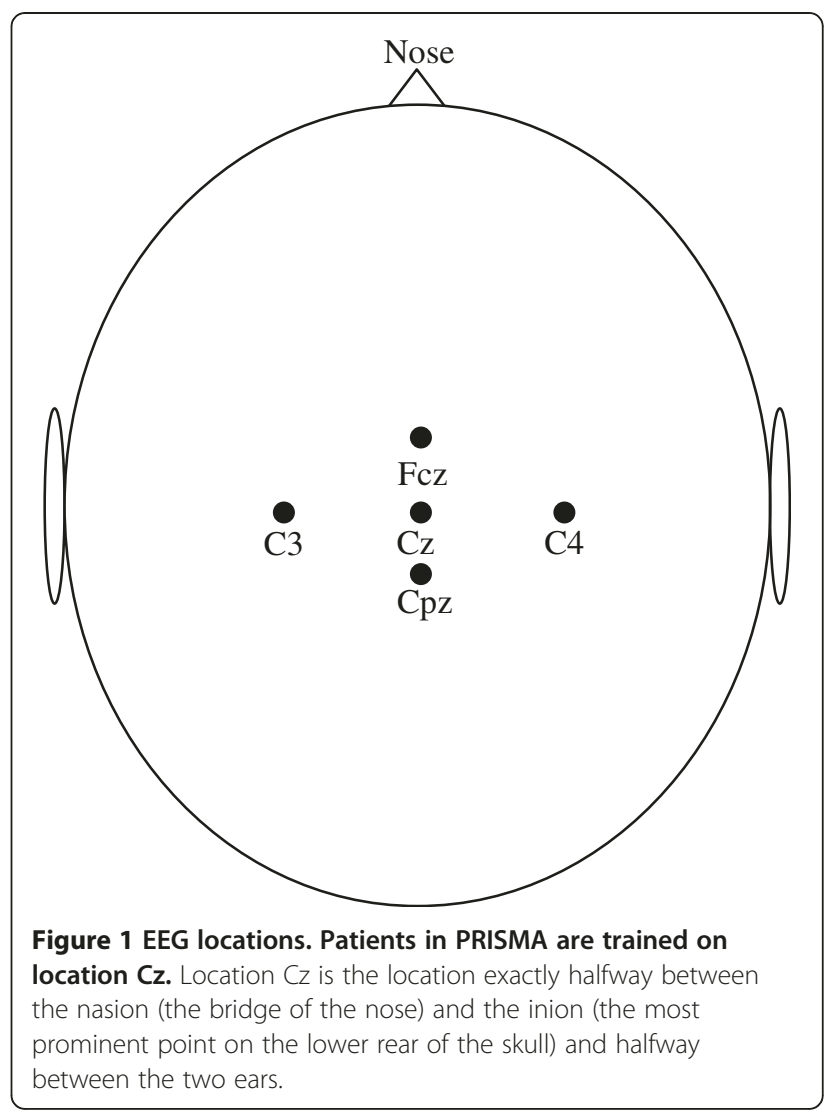

sessions weekly for 15 weeks, 30 sessions in total. Each session takes 39 minutes to administer, divided in ten blocks of three-minutes training, alternated with oneminute breaks. In the breaks the patient will be instructed to sit quietly with the eyes closed.

The neurofeedback sessions are hosted by extensively trained research assistants who have successfully completed a full day schooling session on administration of the neurofeedback training in accordance with detailed standard operating procedures. During the first neurofeedback session, the research assistant will be accompanied by one of the researchers to ensure adherence to the standard operating procedures. After each session, the research assistant is required to fill out a checklist providing information about the training that includes items on start and finish time of the training, duration of the session, selected movie, alertness of the patient, and any deviations from the standard procedures. Checklists are e-mailed to the researchers on a weekly basis.

\section{Neurofeedback treatment modules}

The neurofeedback treatment modules were developed in the software program BioExplorer. To increase comparability, we decided to develop three standard treatment modules based on the qEEGs from the pilot study 
[24], rather than designing an individualized treatment module for each participant. The three treatment modules are (1) beta 1 up training, (2) sensory motor rhythm (SMR) up/beta 1 down training, and (3) beta 1 down training. The qEEG of the patient determines the most suitable of the three neurofeedback treatment modules. The mean Z-score for the power in the beta 1 frequency band $(15-20 \mathrm{~Hz})$ for the electrodes on locations $\mathrm{Fcz}, \mathrm{Cz}, \mathrm{C} 3, \mathrm{C} 4$ and $\mathrm{Cpz}$ are calculated (see Figure 1). For SMR no Z-scores are provided in the brain resource report. SMR power is calculated and p-values are obtained over the average of 9 electrodes (F3, Fz, F4, C3, Cz, C4, P3, Pz and P4). The beta 1 up training is given if the beta 1 power is within the normal range (within 1 standard deviation from the mean) or lowered (more than 1 standard deviation below the mean). The SMR up/beta 1 down training is chosen if the beta 1 power is elevated (more than 1 standard deviation above the mean) and SMR $(12-15 \mathrm{~Hz})$ is within the normal range $(\mathrm{p}>0.05)$ or lowered $(\mathrm{p}<0.05)$. The beta 1 down training is chosen if the patient has beta spindles, ascertained by an EEG specialist via observation, or if both beta 1 is elevated (more than 1 standard deviation above the mean) and SMR power is elevated $(p<0.05)$. Three identical placebo treatment modules were matched to the three neurofeedback treatment modules; beta 1 up placebo, SMR up/beta 1 down placebo and beta 1 down placebo. In the placebo treatment modules, the provided reward of a movie is not based on the desired brain waves from the patient, but on the 'random signal generator' that is incorporated in the BioExplorer software.

All treatment modules are set at an automatic threshold of $80 \%$ reward. This means that the threshold of reward is regularly adjusted in a way that the child sees the movie approximately $80 \%$ of the time and $20 \%$ of the time the screen of the laptop briefly turns black. Elevated muscular tension and electrical noise from surrounding devices (e.g.

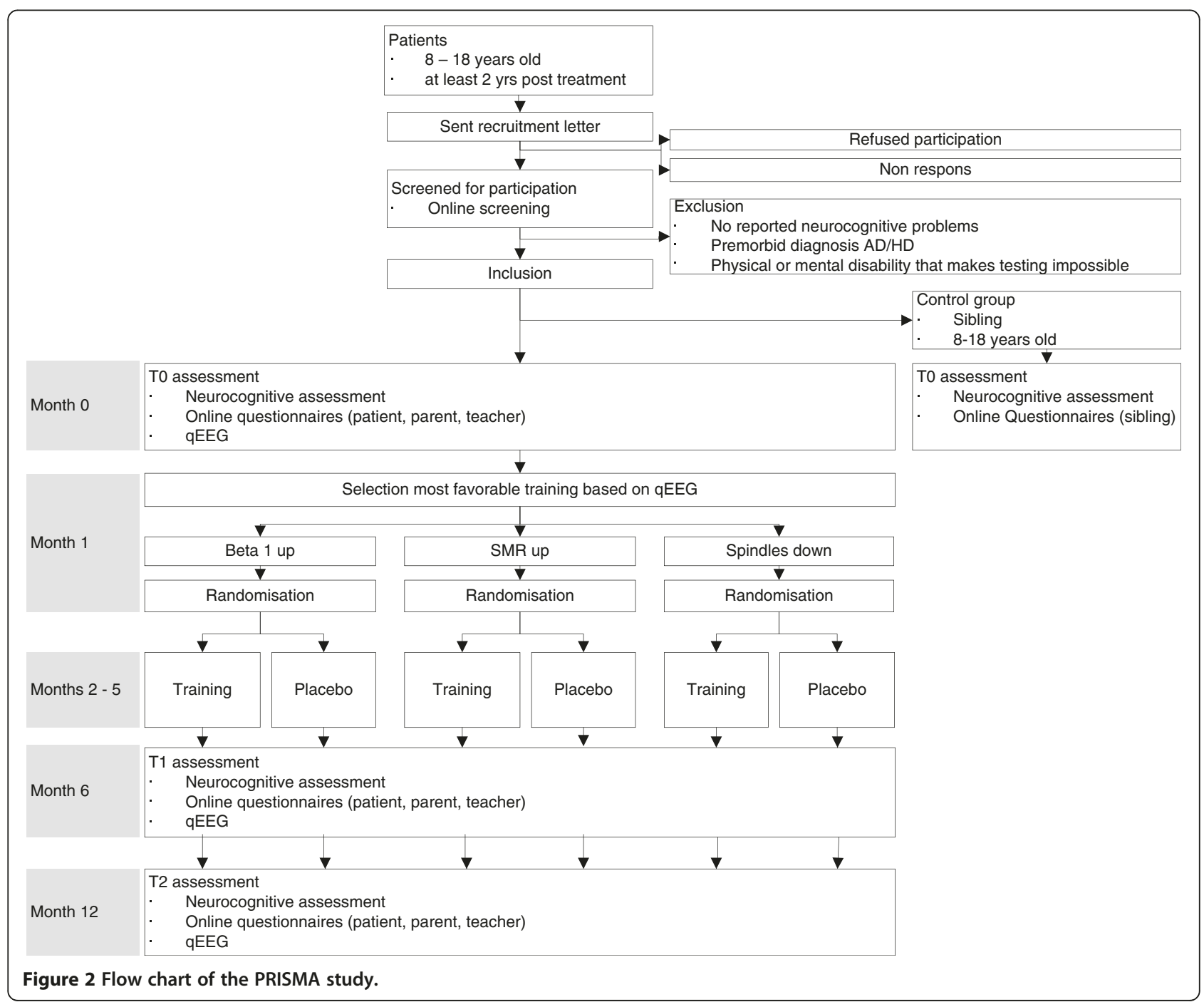


a lamp) can decrease the quality of the training; therefore we employ a threshold of $10 \mu \mathrm{V}$ for muscular tension (> $55 \mathrm{~Hz}$ ) and $50 \mu \mathrm{V}$ for noise (range 48-52 Hz). If the muscular tension and/or the noise reach above the threshold, the movie is interrupted and the computer makes a beeping sound. The training will not continue until the muscular tension and/or noise are brought back under the threshold. This applies to the neurofeedback treatment modules as well as the placebo treatment modules.

\section{Randomization}

The three neurofeedback treatment modules and three placebo treatment modules have been designed to have the exact same appearance on the screen of the notebook, in order to be indistinguishable during training. The six treatment modules have randomly been assigned a number (treatment module 1-6). J.O., one of the members of the research team, holds the key to the codes; the other members are blinded. Another member of the research team analyzes the qEEG and informs J.O. which of the three neurofeedback treatment modules is indicated according to the protocol (see Figure 2). J.O. is then responsible for randomizing the patient into the actual neurofeedback or the placebo training using a randomization table generated by SPSS. For stratfication purposes, randomization takes place after selection of the most appropriate neurofeedback treatment module. After randomization, J.O. notifies M.d. $\mathrm{R}$. of the assigned treatment module (treatment module 1-6) and M.d.R. sends the assigned treatment module via email to the designated research assistant providing the neurofeedback training. Due to his not-blind status, J.O. is not involved in training any patients or the processing of the data.

\section{Procedure \\ Recruitment}

Five out of seven Dutch University hospitals accepted the invitation to join the study. Participating hospitals are the Emma children's hospital/Academic Medical Centre in Amsterdam, VU medical centre in Amsterdam, university medical centre Utrecht in Utrecht, St. Radboud university medical centre in Nijmegen and university medical centre Maastricht in Maastricht. A letter via their oncologist or psychologist informs patients and their caregivers about the study. Interested caregivers will be provided with a screening questionnaire concerning their child's neurocognitive functioning (including items on attention functioning, memory and speed) and exclusion criteria

Table 1 Outcomes, measures and to whom it is administered

\begin{tabular}{|c|c|c|c|c|}
\hline Neurocognitive assessment & Measurement & T0 & T1 & T2 \\
\hline Attention & Attention Network task (ANT) [28] & Patient/sibling (8-18) & Patient (8-18) & Patient (8-18) \\
\hline Processing speed & Baseline Speed ANT [28] & Patient/sibling (8-18) & Patient (8-18) & Patient (8-18) \\
\hline \multirow[t]{2}{*}{ Memory } & Visual Sequencing task & Patient/sibling (8-18) & Patient (8-18) & Patient (8-18) \\
\hline & Digit Span (age appropriate Wechsler scale) $[29,30]$ & Patient/sibling (8-18) & Patient (8-18) & Patient (8-18) \\
\hline \multirow[t]{2}{*}{ Intellectual functioning } & Abbreviated WISC-III* [29] & Patient/sibling (8-16) & - & Patient (8-16) \\
\hline & Abbreviated WAIS-III* [30] & Patient/sibling (17-18) & - & Patient (17-18) \\
\hline Inhibition & Stop Signal task [31] & Patient/sibling (8-18) & Patient (8-18) & Patient (8-18) \\
\hline Visuomotor integration & Tracking and Pursuit task & Patient/sibling (8-18) & Patient (8-18) & Patient (8-18) \\
\hline \multicolumn{5}{|l|}{ Questionnaires } \\
\hline \multirow[t]{3}{*}{ Social/emotional functioning } & SDQ child version [32] & Patient/sibling (8-18) & Patient (8-18) & Patient (8-18) \\
\hline & SDQ parent version [32] & Caregiver (8-18) & Caregiver (8-18) & Caregiver (8-18) \\
\hline & SDQ teacher version [32] & Teacher (8-18) & Teacher (8-18) & Teacher (8-18) \\
\hline \multirow[t]{2}{*}{ Self esteem } & Dutch version SPPC [33] & Patient/sibling (8-11) & Patient (8-11) & Patient (8-11) \\
\hline & Dutch version SPPA [34] & Patient/sibling (12-18) & Patient (12-18) & Patient (12-18) \\
\hline Health related quality of life & Kidscreen 27 [35] & Patient/sibling (8-18) & Patient (8-18) & Patient (8-18) \\
\hline Fatigue & CIS [36] & Patient/sibling (8-18) & Patient (8-18) & Patient (8-18) \\
\hline Sleep disorder & SDSC [37] & Caregiver (8-18) & Caregiver (8-18) & Caregiver (8-18) \\
\hline Attention & SWAN [38] & Caregiver (8-18) & Caregiver (8-18) & Caregiver (8-18) \\
\hline \multirow[t]{2}{*}{ Executive functioning } & BRIEF parent version [39] & Caregiver (8-18) & Caregiver (8-18) & Caregiver (8-18) \\
\hline & BRIEF teacher version [39] & Teacher (8-18) & Teacher (8-18) & Teacher (8-18) \\
\hline
\end{tabular}

Note: Intellectual functioning is assessed at T0 and T2 but not at T1. Siblings are assessed at T0 only.

*The following subtasks were administered: Arythmic, Similarities, Block Design, and Picture Completion.

SDQ = Strengths and Difficulties Questionnaires; SPPC/SPPA = Self Perception Profile for Children/Adolescents; CIS = Checklist Individual Strength; Sleep

Disturbance Scale for Children; SWAN = Strengths and Weaknesses of ADHD-symptoms and Normal-behavior; BRIEF = Behavior Rating Inventory of Executive

Functioning; WISC-III = Wechsler Intelligence Scale for Children - Third version; WAIS-III = Wechsler Adult Intelligence Scale - Third version. 
(e.g. premorbid diagnosis of ADHD or ADD) to verify eligibility of the patient. If eligible for inclusion, the patient is invited for the pre training assessment. If applicable, a sibling will also be invited for assessment to participate in the control group. On the day of the pre training assessment, the informed consent form is signed by caregivers, the patient, and if applicable, the sibling.

\section{Assessment}

Assessments are conducted at one of the three cooperating EEG centers in the Netherlands; Pels institute in Amsterdam, Brainfact in Amsterdam, and EEG resource institute in Nijmegen. Patients are assessed on three occasions: pre training (T0), directly post training (T1), and six months post training (T2); see Figure 2. Assessments include neurocognitive testing, questionnaires filled out by patient, caregiver, and teacher, and a qEEG, see Table 1. Assessment of the siblings occurs only once, and is identical to the assessments used in patients with the exception of questionnaires filled out by parents and teachers.

qEEG An EEG is recorded from the patients at three time points. A Quick-cap with NuAmp 10-20 electrodes international system from neuroscan is used, with 28 channels [40]; Fp1, Fp2, F7, F3, Fz, F4, F8, FC3, FCz, FC4, T3, C3, Cz, C4, T4, CP3, CPz, CP4, T5, P3, Pz, P4, $\mathrm{T} 6, \mathrm{O} 1, \mathrm{Oz}$ and $\mathrm{O} 2$. During the first three minutes an eyes-open resting EEG is registered, in the consecutive three minutes an eyes-closed resting EEG. After the resting EEG, event related potentials are measured during an odd ball and a go-nogo task. The Brain Resource International Database (BRID) [41,42], comprising EEG power spectra of over 4.000 healthy controls, provides normative data to quantify the EEG (qEEG) and obtain Z-scores for the participants in the current study.

Neurocognitive tests To objectify the primary hypothesis of the study, that neurofeedback will improve neurocognitive functioning, different neurocognitive domains are assessed. The tests are conducted by one of the researchers or extensively trained research assistants and take approximately two and a half hours. Based on literature describing late effects in brain tumor patients, the following neurocognitive domains were targeted for assessment [43]: attention, processing speed, memory, intellectual functioning, inhibition, and visuomotor integration. Well-validated computerized and pencil-and-paper tests were selected to provide a comprehensive assessment of neurocognitive functioning before the training and the efficacy of neurofeedback (see Table 1).

Questionnaires Our secondary hypothesis regards the impact of neuropsychological performance on psychosocial functioning $[25,44]$. Based on studies reports, the following domains are assessed using questionnaires: social/emotional functioning, self-esteem, and health related quality of life. Because of the reported decrease in fatigue after training in the pilot study, we also included questionnaires assessing fatigue and sleep disturbance [24]. In addition, two questionnaires on attention and executive functioning were added to assess caregiver and teacher rated neurocognitive functioning. Widely used, reliable, and validated questionnaires were selected in order to assess the domains of interest, as well as the effect of neurofeedback on these domains (see Table 1). Questionnaires were administered to either patient, caregivers or teacher, if applicable. The online questionnaires take approximately 30 minutes to fill out. In addition, as an interim measurement, caregivers fill in the attention questionnaire (SWAN [44]) one extra time, after the first 10 sessions of the patient.

\section{Power calculation}

Power calculations used the neurocognitive measures as primary outcome measures. The calculations were done in the statistical program nQuery Advisor [45]. We expect that the neurofeedback will have a medium $(\mathrm{d}=0.5)$ to large effect $(\mathrm{d}=0.8)$ on neurocognitive functioning as measured at T1 and compared to T0, based on improvements found in children with ADHD who were trained with neurofeedback and on the improvements found in learning-impaired childhood cancer survivors treated with methylphenidate $[12,15]$. Given an effect size of 0.6 with alpha set at 0.05 (one-sided) and a power of 0.80 , a minimum of 35 patients is required in both the neurofeedback group and the placebo group.

\section{Statistical analyses}

Intention-to-treat analyses will be conducted. Because of possible withdrawal before treatment starts, dropouts during the study, failure to fill out questionnaires, or research procedure violations, missing data will occur. Imputation of missing values will be carried out as much as possible to make intention-to-treat analyses feasible. Missing data will be imputed using Imputation and Variance Estimation Software [46].

Prior to the training (T0) we will assess differences between patients and siblings on neurocognitive and psychosocial functioning, using mixed modelling. Subsequently, we will conduct multivariate analysis of variance (MANOVA) to determine the effect of neurofeedback post-treatment (T1) on the primary and secondary outcomes, comparing the patients in the neurofeedback group to the patients in the placebo group. To control for possible differences in neurocognitive functioning prior to the training, T0 data will be included in the model as covariate. Finally, we will use 
repeated measures analysis for group (neurofeedback and placebo) $\mathrm{x}$ time (T0, T1, and six months follow-up, T2) to investigate the changes over time. To examine the possible effects of patient characteristics on the efficacy of the neurofeedback, the following variables will be assessed as covariates in the MANOVA and repeated measures analyses: age at assessment, age at diagnosis, diagnosis, time since diagnosis, and treatment modalities. All analyses will be conducted using SPSS. A $P$-value $<0.05$ will be considered significant.

\section{Discussion}

This article describes the design of the PRISMA study, a randomized controlled trial investigating the efficacy of neurofeedback in pediatric brain tumor survivors with neurocognitive problems. Although neurocognitive problems in pediatric oncology survivors are reported in numerous studies, empirically validated interventions addressing these deficits are scarce. There is growing evidence for neurofeedback as a valuable treatment in different brain disorders $[15,21]$. Our study is the first to investigate the efficacy of neurofeedback in pediatric brain tumor survivors using a randomized placebocontrolled double-blind trial, comparing neurofeedback to placebo training. By setting an automatically adjusted threshold of feedback as opposed to a manually adjusted threshold, we enabled blinding the trainers; trainers were not required to monitor the brain activity of the patient during the sessions. Furthermore, we ensure the standardization of the neurofeedback treatment by employing carefully instructed research assistants providing the neurofeedback treatment. At the same time we increased the feasibility for the patients, by administering the training at the patients' home or school. In addition, the effects of neurofeedback on neurocognitive and psychosocial functioning are thoroughly investigated by using well-validated paradigms and psychometrically sound questionnaires administered to patient, caregiver and teacher. Lastly, we have included a control group of healthy siblings, to compare performance of the brain tumor survivors to children without a history of a brain tumor.

The design of PRISMA has some methodological pitfalls to take into account. Because of time factors and the population, this study might be at risk for losing patients during the treatment phase and during followup. With five year survival rates of approximately $65 \%$, some patients may relapse [47], or they may discontinue their participation in the study. We increase comparability by employing three different training modules; however, the training might be less effective than an individualized training. The groups receiving each of the three treatment modules are small. Also, the group of brain tumor patients is heterogeneous, e.g. in terms of tumor diagnosis, tumor location, age at diagnosis, treatment, time since diagnosis, and time since end of treatment. It is well documented that these variables play an important role in neurocognitive outcomes. These heterogeneities may be reflected in our results.

In conclusion, if neurofeedback proves to be effective in improving neurocognitive deficits after treatment for a brain tumor, this would be a valuable addition to the currently available effective interventions for this vulnerable group of pediatric brain tumor survivors.

\section{Competing interest}

The authors have no financial relationship or conflicts of interest to disclose.

\section{Authors' contribution}

MdR, AS, RvM, JG, JO and MG made substantial contributions to conception and design of the study. MdR, AS, RvM, TJ, JG, JO and MG helped drafting the article or revising it critically for important intellectual content. All authors read and approved the final manuscript.

\section{Author details}

${ }^{1}$ Psychosocial Department, Emma Children's Hospital AMC, room A3-241, Meibergdreef 9, Amsterdam 1105 AZ, The Netherlands. 'Pediatric Oncology, Emma Children's Hospital AMC, room G8-236, Meibergdreef 9, Amsterdam 1105 AZ, The Netherlands. ${ }^{3} \mathrm{VU}$ University Amsterdam, Van der Boechorststraat 1, room 1E-41, Amsterdam 1081 BT, The Netherlands.

Received: 4 October 2012 Accepted: 22 November 2012

Published: 6 December 2012

\section{References}

1. Mariotto AB, Rowland JH, Yabroff KR, Scoppa S, Hachey M, Ries L, Feuer EJ: Long-term survivors of childhood cancers in the United States. Canc Epidemiol 2009, 18:1033-1040

2. Mabbott D, Penkman L, Witol A: Core neurocognitive functions in children treated for posterior fossa tumors. Neuropsychology 2008, 22:159-168.

3. Mulhern RK, Palmer SL, Merchant TE, Wallace D, Kocak M, Brouwers P, Krull K, Chintagumpala M, Stargatt R, Ashley DM, Tyc VL, Kun L, Boyett J, Gajjar A: Neurocognitive consequences of risk-adapted therapy for childhood medulloblastoma. J Clin Oncol 2005, 23:5511-5519.

4. Zebrack BJ, Gurney JG, Oeffinger K, Whitton J, Packer RJ, Mertens A, Turk N, Castleberry R, Dreyer Z, Robison LL, Zeltzer LK: Psychological outcomes in long-term survivors of childhood brain cancer: a report from the childhood cancer survivor study. J Clin Oncol 2004, 22:999-1006.

5. Duffner PK: Risk factors for cognitive decline in children treated for brain tumors. Eur J Paediatr Neurol 2010, 14:106-115.

6. De Ruiter MA, Van Mourik R, Schouten-Van Meeteren AYN, Grootenhuis MA, Oosterlaan J: Neurocognitive consequences of a pediatric brain tumor and its treatment: a meta-analysis. Developmental medicine and child neurology (accepted), 2012

7. Carey ME, Barakat LP, Foley B, Gyato K, Phillips PC: Neuropsychological functioning and social functioning of survivors of pediatric brain tumors: evidence of nonverbal learning disability. Child Neuropsychology 2002, 7(4):265-272.

8. Butler RW, Mulhern RK: Neurocognitive interventions for children and adolescents surviving cancer. J Pediatr Psychol 2005, 30:65-78.

9. Butler RW, Copeland DR, Fairclough DL, Mulhern RK, Katz ER, Kazak AE, Noll RB, Patel SK, Sahler OJ: A multicenter, randomized clinical trial of a cognitive remediation program for childhood survivors of a pediatric malignancy. J Consul Clin 2008, 76:367-378.

10. Van't Hooft I, Andersson K, Bergman B, Sejersen T, Von Wendt L, Bartfai A: Sustained favorable effects of cognitive training in children with acquired brain injuries. NeuroRehabilitation 2007, 22:109-116.

11. Mulhern RK, White HA, Glass JO, Kun LE, Leigh L, Thompson SJ, Reddick WE: Attentional functioning and white matter integrity among survivors of malignant brain tumors of childhood. J Int Neuropsychol Soc 2004, 10:180-189. 
12. Thompson SJ, Leigh L, Christensen R, Xiong X, Kun LE, Heideman RL, Reddick WE, Gajjar A, Merchant T, Pui CH, Hudson MM, Mulhern RK: Immediate neurocognitive effects of methylphenidate on learning-impaired survivors of childhood cancer. J Clin Oncol 2001, 19:1802-1808.

13. Simonoff E, Taylor E, Baird G, Bernard S, Chadwick O, Liang H, Whitwell S, Riemer K, Sharma K, Sharma SP, Wood N, Kelly J, Golaszewski A, Kennedy J, Rodney L, West N, Walwyn R, Jichi F: Randomized controlled double-blind trial of optimal dose methylphenidate in children and adolescents with severe attention deficit hyperactivity disorder and intellectual disability. J Child Psychol Psychiatry 2012.

14. Sterman M: Basic concepts and clinical findings in the treatment of seizure disorders with EEG operant conditioning. Clin Electroencephalogr 2000, 31:45-55.

15. Heinrich H, Gevensleben H, Strehl U: Annotation: neurofeedback - train your brain to train behaviour. J Child Psychol Psychiatry 2007, 48:3-16.

16. Hirshberg LM, Chiu S, Frazier JA: Emerging brain-based interventions for children and adolescents: overview and clinical perspective. Child Adolesc Psychiatr Clin N Am 2005, 14:1-19.

17. Arns M, Ridder SD, Strehl U, Breteler M, Coenen A: Efficacy of neurofeedback treatment in ADHD: the effects on inattention, impulsivity and hyperactivity: a meta-analysis. Clinical Eeg And Neuroscience 2009, 40:180-189.

18. Hodgson K, Hutchinson AD, Denson L: Nonpharmacological treatments for ADHD: a meta-analytic review. J Atten Disord 2012, XX:1-8.

19. Gevensleben $H$, Rothenberger $A$, Moll GH, Heinrich $H$ : Neurofeedback in children with ADHD: validation and challenges. Expert Rev Neurother 2012, 12:447-460.

20. Strehl U, Leins $U$, Goth $G$, Klinger $C$, Hinterberger T, Birbaumer N: Self-regulation of slow cortical potentials: a new treatment for children with attention-deficit/hyperactivity disorder. Pediatrics 2006, 118:e1530-e1540.

21. Gevensleben H, Holl B, Albrecht B, Schlamp D, Kratz O, Studer $P$, Rothenberger A, Moll GH, Heinrich H: Neurofeedback training in children with ADHD: 6-month follow-up of a randomised controlled trial. Eur Child Adolesc Psychiatry 2010, 19:715-724.

22. Lofthouse N, Arnold LE, Hersch S, Hurt E, DeBeus R: A review of neurofeedback treatment for pediatric ADHD. J Atten Disord 2012, 16:351-372.

23. Thornton KE, Carmody DP: Electroencephalogram biofeedback for reading disability and traumatic brain injury. Child Adolesc Psychiatr Clin N Am 2005, 14:137-162. vii.

24. Aukema EJ, Schouten-van Meeteren AY, Last BF, Breteler MHM, Hogeweg J, Grootenhuis MA: Exploring the feasibility of neurofeedback training as a cognitive intervention for childhood brain tumor survivors: a pilot study. Submitted.

25. Schulte F, Barrera M: Social competence in childhood brain tumor survivors: a comprehensive review. Support Care Cancer 2010, 18:1499-1513.

26. BioExplorer. http://www.cyberevolution.com/.

27. BrainQuiry. http://www.brainquiry.com/.

28. Fan J, McCandliss BD, Sommer T, Raz A, Posner Ml: Testing the efficiency and independence of attentional networks. J Cogn Neurosci 2002, 14:340-347.

29. Wechsler D: Wechsler's Intelligence scale for children. 3rd edition. London: Harcourt Brace and Company; 1992.

30. Wechsler D: Wechsler adult intelligence scale-III (WAIS-III). San Antonio, TX: The Psychological Corporation; 1997.

31. Logan GD, Cowan WB: On the ability to inhibit thought and action: a theory of an act of control. Psychol Rev 1984, 91:295-327.

32. Goodman $R$ : The strengths and difficulties questionnaire: a research note. J Child Psychol Psychiatry 1997, 38:581-586.

33. Veerman JW, Straathof MAE, Treffers PDA, Van den Bergh BRH, Ten BL: Handleiding bij de competentie belevingsschaal voor kinderen, CBSK (manual of the self perception profile for children - Dutch version). Amsterdam, Lisse: Swets \& Zeitlinger; 1997

34. Treffers PDA, Goedhart AW, Veerman JW, Van den Bergh BRH, Ackaert L, De Rycke L: Handleiding bij de competentie belevingsschaal voor adolescenten, CBSA (manual of the self perception profile for adolescents - dutch version). Amsterdam, Lisse: Swets \& Zeitlinger; 2002.
35. Ravens-Sieberer U, Auquier P, Erhart M, Gosch A, Rajmil L, Bruil J, Power M, Duer W, Cloetta B, Czemy L, Mazur J, Czimbalmos A, Tountas Y, Hagquist C, Kilroe J: The KIDSCREEN-27 quality of life measure for children and adolescents: psychometric results from a cross-cultural survey in 13 European countries. Qual Life Res 2007, 16:1347-1356.

36. Stulemeijer M, De Jong LWA M, Fiselier TJW, Hoogveld SWB, Bleijenberg G: Cognitive behaviour therapy for adolescents with chronic fatigue syndrome: randomised controlled trial. BMJ (Clinical research ed.) 2005, 330:14.

37. Bruni O, Ottaviano S, Guidetti V, Romoli M, Innocenzi M, Cortesi F, Giannotti F: The sleep disturbance scale for children (SDSC). construction and validation of an instrument to evaluate sleep disturbances in childhood and adolescence. J Sleep Res 1996, 5:251-261.

38. Polderman TJC, Derks EM, Hudziak JJ, Verhulst FC, Posthuma D, Boomsma DI: Across the continuum of attention skills: a twin study of the SWAN ADHD rating scale. J Child Psychol Psychiatry 2007, 48:1080-1087.

39. Gioia GA, Isquith PK, Guy SC, Kenworthy L: TEST REVIEW behavior rating inventory of executive function behavior rating inventory of executive function. Psychol Assess 2000, 6(3):235-238.

40. Neuroscan. http://www.neuroscan.com/

41. Gordon E, Cooper N, Rennie C, Hermens D, Williams LM: Integrative neuroscience: the role of a standardized database. Clin EEG Neurosci 2005, 36:64-75.

42. Brain resource. http://www.brainresource.com/.

43. Mulhern RK, Merchant TE, Gajjar A, Reddick WE, Kun LE: Late neurocognitive sequelae in survivors of brain tumours in childhood. Lancet Oncol 2004, 5:399-408.

44. Bhat SR, Goodwin TL, Burwinkle TM, Lansdale MF, Dahl GV, Huhn SL, Gibbs IC, Donaldson SS, Rosenblum RK, Varni JW, Fisher PG: Profile of daily life in children with brain tumors: an assessment of health-related quality of life. J Clin Oncol 2005, 23:5493-5500.

45. Elashoff JD: NQuery advisor version 7.0 User's Guide. Los Angeles, CA: 2007

46. IVEware: Imputation and variance estimation software. http://www.isr.umich.edu/src/smp/ive/.

47. Kaatsch P: Epidemiology of childhood cancer. Cancer Treat Rev 2010, 36:277-285.

doi:10.1186/1471-2407-12-581

Cite this article as: de Ruiter et al:: Neurofeedback to improve neurocognitive functioning of children treated for a brain tumor: design of a randomized controlled double-blind trial. BMC Cancer 2012 12:581.

\section{Submit your next manuscript to BioMed Central and take full advantage of:}

- Convenient online submission

- Thorough peer review

- No space constraints or color figure charges

- Immediate publication on acceptance

- Inclusion in PubMed, CAS, Scopus and Google Scholar

- Research which is freely available for redistribution 